\title{
AN APPLICATION OF FLOWS TO TIME SHIFT AND TIME REVERSAL IN STOCHASTIC PROCESSES ${ }^{1}$
}

\author{
BY
}

E. B. DYNKIN

\begin{abstract}
A simple proposition (Theorem 1) on flows allows the investigation of random time shift and time reversal in Markov processes without assuming any regularity of paths. Theorem 5 is a generalization of Nagasawa's time reversal theorem and Theorem 4 generalizes a recent result of Getoor and Glover.
\end{abstract}

\section{Flows.}

1.1. We consider a flow in a measurable space $(\Omega, \mathscr{F})$ that is a family of transformations $\theta_{t}, t \in \mathbf{R}$, such that $\theta_{s} \theta_{t}=\theta_{s+t}$ for all $s, t \in \mathbf{R}$ and $\left\{(t, \omega): \theta_{t} \omega \in A\right\}$ $\in \mathscr{B}_{\mathbf{R}} \times \mathscr{B}$ for all $A \in \mathscr{F}\left(\mathscr{B}_{R}\right.$ is the Borel $\sigma$-algebra in $\left.\mathbf{R}\right)$.

We put $\left(\theta_{t} Y\right)(\omega)=Y\left(\theta_{t} \omega\right)$, for every function $Y$ and

$$
\begin{gathered}
\left(P \theta_{t}\right)(A)=P\left(\theta_{t}^{-1} A\right), \quad A \in \mathscr{F}, \\
\bar{P}=\int_{\mathbf{R}} P \theta_{t} d t,
\end{gathered}
$$

for every measure $P$. We denote by $\mathscr{A}$ the collection of all sets $A \in \mathscr{F}$ such that $\theta_{t}^{-1} A=A$ for all $t \in \mathbf{R}$. A function $Y$ is measurable with respect to the $\sigma$-algebra $\mathscr{A}$ if and only if it is $\mathscr{F}$-measurable and invariant under all transformations $\boldsymbol{\theta}_{\boldsymbol{t}}$. Obviously $\bar{P}(A)=0$ or $+\infty$ for all $A \in \mathscr{A}$. Nevertheless we prove

THEOREM 1. If $\bar{P}$ is $\sigma$-finite (over $\mathscr{F}$ ), it determines uniquely the values $P(A)$ for all $A \in \mathscr{A}$.

1.2. As a tool we use stationary times. A stationary time $\tau$ is a measurable mapping from $\Omega$ to the extended real line $[-\infty,+\infty]$ such that $\theta_{t} \tau=\tau-t$ for all $t \in \mathbf{R}$.

Suppose that $\mathscr{F}$ coincides with its completion with respect to the class of all probability measures. Then the first hitting time of a set $A \in \mathscr{F}$,

$$
\tau_{A}^{+}=\inf \left\{t: \theta_{t} \omega \in A\right\},
$$

and the last exit time from $A$,

$$
\tau_{A}^{-}=\sup \left\{t: \theta_{t} \omega \in A\right\}
$$

are stationary times $^{2}$ (the measurability follows from [5, Chapter $\left.3, \S 1\right]$ ).

Received by the editors March 13, 1984.

1980 Mathematics Subject Classification. Primary 60G, 60I.

${ }^{1}$ Supported in part ;by National Science Foundation Grant MCS-8202286.

${ }^{2}$ We put inf $\phi=+\infty, \sup \phi=-\infty$. 
In general, every stationary time $\tau$ is the first hitting time of $\{\tau<0\}$ and it is the last exit time from $\{\tau>0\}$.

We denote the indicator function for the set $\{\tau \in \mathbf{R}\}$ by $\kappa_{\tau}$. Let $\rho$ be a positive Borel function on $\mathbf{R}$ such that $\int \rho(t) d t=1$. We put $\rho(-\infty)=\rho(+\infty)=0$. We note that $\theta_{t} \rho(\tau)=\rho(\tau-t)$. Hence

$$
\kappa_{\tau}=\int \rho(\tau-t) d t=\int \theta_{t} \rho(\tau) d t
$$

and, for every $\mathscr{A}$-measurable $Y$,

$$
P \kappa_{\tau} Y=P Y \int \theta_{t} \rho(\tau) d t=\int P \theta_{t}(Y \rho(\tau))=\bar{P} \rho(\tau) Y
$$

1.3. Suppose that there exists a strictly positive function $F$ such that

$$
F_{s}=\int_{-\infty}^{s} \theta_{t} F d t \quad \text { is } P \text {-integrable for some } s \in \mathbf{R} .
$$

Put

$$
\tau_{a}=\inf \left\{s: F_{s} \geqslant a\right\}, \quad a>0 .
$$

Note that $P\left\{\tau_{a}=-\infty\right\}=0$ and $\left\{\tau_{a}=+\infty\right\}=\left\{F_{\infty} \leqslant a\right\}$. Hence $\kappa_{\tau_{a}}=1_{F_{\infty}>a} \uparrow 1$ $P$-a.s. as $a \downarrow 0$. By (1.1),

$$
P Y=\lim _{a \downarrow 0} \bar{P} Y \rho\left(\tau_{a}\right) .
$$

The restriction of measure $P$ to $\mathscr{A}$ can be recovered from $\bar{P}$ by formula (1.3). An analogous formula can be written if

$$
F^{s}=\int_{s}^{+\infty} \theta_{t} F d t \quad \text { is } P \text {-integrable for some } s \in \mathbf{R} \text {. }
$$

Both conditions (1.2) and (1.4) are satisfied if $\bar{P}$ is $\sigma$-finite.

1.4. Let $\tau$ be a stationary time. For every function $Z(\omega)$ we denote by $\theta_{\tau} Z$ the function $Y(\omega)$ defined by the formula

$$
Y(\omega)= \begin{cases}Z\left(\theta_{\tau(\omega)} \omega\right) & \text { if } \tau(\omega) \in \mathbf{R}, \\ 0 & \text { otherwise. }\end{cases}
$$

It is clear that $Y$ is invariant and therefore formulas (1.1) and (1.3) are applicable to $Y$. We conclude

If $P_{1}$ and $P_{2}$ are two measures on $(\Omega, \mathscr{F})$ and if $\bar{P}_{1}=\bar{P}_{2}$ is a $\sigma$-finite measure, then $P_{1} \theta_{\tau}=P_{2} \theta_{\tau}$ for all stationary times $\tau$.

1.5. The definition of the measure $\bar{P}$ makes sense if an arbitrary locally compact group $G$ acts on $(\Omega, \mathscr{F})$ (the Lebesgue measure on $\mathbf{R}$ should be replaced by a left- or right-invariant measure on $G$ ). Formula (1.1) and its proof remain valid if $\tau$ is a $G$-valued function defined on an invariant set $\Omega_{\tau}$ such that $\theta_{g} \tau(\omega)=\tau(\omega) g^{-1}$ for all $g \in G$. (We put $\kappa_{\tau}=1_{\Omega_{\tau}}$ and we set $\rho(\tau)=0$ if $\tau(\omega)$ is not defined.)

However the construction in subsection 1.3 is not applicable if $G \neq \mathbf{R}$. It remains an open problem for which groups $G$ Theorem 1 is true. 


\section{Random time shift in Markov processes.}

2.1. Let $p(s, x ; t, B)$ be a Markov transition function in $(E, \mathscr{E})$. We put

$$
\mu T_{t}^{s}(B)=\int \mu(d x) p(s, x ; t, B) ; \quad T_{t}^{s} f(x)=\int p(s, x ; t, d y) f(y) .
$$

Suppose that a $\sigma$-finite measure $\nu_{t}$ is given for every $t \in \mathbf{R}$. We say that $\nu=\left\{\nu_{t}\right\}$ is an entrance rule if, for all $B \in \mathscr{E}$,

$$
\nu_{s} T_{t}^{s}(B) \leqslant \nu_{t}(B) \quad \text { and } \quad \nu_{s} T_{t}^{s}(B) \uparrow \nu_{t}(B) \quad \text { as } s \uparrow t .
$$

$\nu$ is an entrance rule at time $r$ if $\nu_{t}=0$ for $t \leqslant r$ and $\nu_{s} T_{t}^{s}=\nu_{t}$ for $r<s<t$. $^{3}$ In an analogous way, we define an entrance rule at time $-\infty$. Every entrance rule has a representation

$$
\nu=\int_{[-\infty,+\infty]} \nu^{r} \sigma(d r)
$$

where $\nu^{r}$ is an entrance rule at time $r$. We note that $\sigma\{-\infty\}=0$ if and only if $\nu_{s} T_{t}^{s} \downarrow 0$ as $t \uparrow \infty$ for some $s$ (see [1, Formula (5.5)]).

A family $h=\left\{h^{t}, t \in \mathbf{R}\right\}$ of positive $\mathscr{E}$-measurable functions is called an exit rule if

$$
T_{t}^{s} h^{t} \leqslant h^{s} \quad \text { and } \quad T_{t}^{s} h^{t} \uparrow h^{s} \quad \text { as } t \downarrow s .
$$

All concepts related to entrance rules have natural analogs for exit rules.

The following result is due to Kuznetsov [4]. Suppose that $(E, \mathscr{E})$ is a standard Borel space and let an entrance rule $\mu$ and an exit rule $h$ satisfy the condition

$$
\mu_{t}\left(h^{t}=+\infty\right)=0 \text { for all } t .
$$

Then there exists a stochastic process $\left(X_{t}, P_{\mu}^{h}\right)$ on a random time interval $(\alpha, \beta)$ such that, for every $t \in \mathbf{R}$,

$$
P_{\mu}^{h}\left(\alpha<t, X_{t} \in d y, t<\beta\right)=\mu_{t}(d y) h^{t}(y),
$$

and for all $t_{1}<\cdots<t_{n} \in \mathbf{R}$,

$$
\begin{aligned}
P_{\mu}^{h}(\alpha & \left.<t_{1}, X_{t_{1}} \in d y_{1}, \ldots, X_{t_{n}} \in d y_{n}, t_{n}<\beta\right) \\
& =\mu_{t_{1}}\left(d y_{1}\right) p\left(t_{1}, y_{1} ; t_{2}, d y_{2}\right) \cdots p\left(t_{n-1}, y_{n-1} ; t_{n}, d y_{n}\right) h^{t_{n}}\left(y_{n}\right) .
\end{aligned}
$$

The measure $P_{\mu}^{h}$ is $\sigma$-finite.

2.2. Suppose that a transition function $p$ is stationary. Excessive measures and functions can be characterized as entrance and exit rules independent of $t$. Let $\mu$ be an entrance rule. If the measure

$$
\bar{\mu}=\int_{\mathbf{R}} \mu_{t} d t
$$

is $\sigma$-finite, it is excessive. To every exit rule $h$ there corresponds an excessive function

$$
\bar{h}=\int_{\mathbf{R}} h^{t} d t
$$

\footnotetext{
${ }^{3}$ In the literature, entrance rules at time 0 are called entrance laws.
} 
We assume that the process $X_{t}(\omega)$ can be chosen in such a way that there exists a flow $\theta_{t}, t \in \mathbf{R}$, with the properties: $\theta_{t} \alpha=\alpha-t, \theta_{t} \beta=\beta-t$ and $\theta_{t} X_{u}=X_{u+t}{ }^{4}$

$A$ random time shift of the process $X_{t}$ is defined on the space $\Omega_{\tau}=\{\omega:-\infty<\tau$ $<+\infty$ ) by the formula

$$
\tilde{X}_{t}(\omega)=X_{\tau(\omega)+t}(\omega) .
$$

Suppose that an entrance rule $\mu$ and an exit rule $h$ satisfy the conditions

$$
\bar{\mu} \text { is } \sigma \text {-finite and } \bar{h}<\infty \quad \bar{\mu} \text {-a.e. }
$$

Then the conditions of Kuznetsov's theorem are satisfied for three pairs $(\bar{\mu}, \bar{h})$, $(\bar{\mu}, h)$ and $(\mu, \bar{h})$ and therefore, the measures $P_{\bar{\mu}}^{\bar{h}}, P_{\bar{\mu}}^{h}$ and $P_{\mu}^{\bar{h}}$ are defined and $\sigma$-finite. We note that

$$
\overline{P_{\bar{\mu}}^{h}}=\overline{P_{\mu}^{\bar{h}}}=P_{\bar{\mu}}^{\bar{h}} .
$$

By applying Theorem 1 and subsection 1.4, we get the following result:

THEOREM 2. Measures $P_{\bar{\mu}}^{h}$ and $P_{\mu}^{\bar{h}}$ coincide on all invariant sets. If $\tau$ is a stationary time, then

$$
P_{\bar{\mu}}^{h} \theta_{\tau}=P_{\mu}^{\bar{h}} \theta_{\tau} .
$$

The shift $\tilde{X}_{t}$ of $X_{t}$ defined by formula (2.5) has identical laws under $P_{\bar{\mu}}^{h}$ and $P_{\mu}^{\bar{h}}$.

3. Backward transition functions and duality. Theorem of Nagasawa and theorem of Getoor and Glover.

3.1. Along with forward transition functions $p(s, x ; t, d y)$ we consider backward transition functions $q(s, d x ; t, y)$. We say that a forward transition function $p$ and a backward transition function $q$ are m-related if

$$
m_{s}(d x) p(s, x ; t, d y)=q(s, d x ; t, y) m_{t}(d y) \text { for all } s<t .
$$

It follows from this relation that $m$ is an entrance rule for both $p$ and $q$.

If $g=\left\{g_{t}\right\}$ is an exit rule for $q$, then $(g \circ m)_{t}(d x)=g_{t}(x) m_{t}(d x)$ is an entrance rule for $p$, and all entrance rules for $p$ which are absolutely continuous with respect to $m$ can be represented in this form.

To every statement on forward transition functions there corresponds a statement on backward transition functions. The "backward" version of Kuznetsov's theorem is as follows. Let $\nu=\left\{\nu^{t}\right\}$ be an entrance rule and $g=\left\{g_{t}\right\}$ be an exit rule for a backward transition function $q$. If

$$
\nu^{t}\left(g_{t}=+\infty\right)=0 \text { for all } t
$$

then there exists a stochastic process $\left(X_{t}, Q_{g}^{\nu}\right)$ on a random time interval $(\alpha, \beta)$ such that, for every $t \in \mathbf{R}$,

$$
Q_{g}^{\nu}\left(\alpha<t, X_{t} \in d y, t<\beta\right)=g_{t}(y) \nu^{t}(d y)
$$

\footnotetext{
${ }^{4}$ (The $\sigma$-algebra $\mathscr{F}$ in $\Omega$ is generated by the sets $\left\{X_{t} \in B\right\}, t \in \mathbf{R}, B \in \mathscr{E}$.) Counterexamples show that Theorems 2 through 5 are not true without this assumption.
} 
and, for all $t_{1}<\cdots<t_{n} \in \mathbf{R}$,

$$
\begin{aligned}
Q_{g}^{\nu}(\alpha & \left.<t_{1}, X_{t_{1}} \in d y_{1}, \ldots, X_{t_{n}} \in d y_{n}, t_{n}<\beta\right) \\
& =g_{t_{1}}\left(y_{1}\right) q\left(t_{1}, d y_{1} ; t_{2}, y_{2}\right) \cdots q\left(t_{n-1}, d y_{n-1} ; t_{n}, y_{n}\right) \nu^{t_{n}}\left(d y_{n}\right) .
\end{aligned}
$$

The measure $Q_{g}^{\nu}$ is $\sigma$-finite.

Suppose that $p$ and $q$ are $m$-related and that $h$ is an exit rule for $p$ and $g$ is an exit rule for $q$. It follows from (3.3) and (3.4) that

$$
P_{g \circ m}^{h}=Q_{g}^{h \circ m} \text {. }
$$

3.2. If a backward transition function $q$ is stationary, then we write

$$
q_{t}(d x, y)=q(t, d x ; 0, y), \quad t<0 .
$$

If $p$ and $q$ are both stationary and if $m_{t}=m$ for all $t$, then the condition (3.1) can be rewritten as follows

$$
m(d x) p_{t}(x, d y)=q_{-t}(d x, y) m(d y) .
$$

Suppose that $\mu$ is an entrance rule for $p, \nu$ is an entrance rule for $q$ and let $\bar{\mu}$ and $\bar{\nu}$ be $\sigma$-finite and absolutely continuous with respect to $m$. The densities $u=d \bar{\mu} / d m$ and $v=d \bar{\nu} / d m$ can be chosen to be $q$-excessive and $p$-excessive respectively. By (3.5),

$$
\overline{P_{\mu}^{v}}=P_{\bar{\mu}}^{v}=P_{u \circ m}^{v}=Q_{u}^{v \circ m}=Q_{u}^{\bar{\nu}}=\overline{Q_{u}^{\nu}},
$$

and Theorem 1 and subsection 1.4 imply

THEOREM 3. Measures $P_{\mu}^{v}$ and $Q_{u}^{\nu}$ coincide on all invariant sets. For every stationary time $\tau, P_{\mu}^{v} \theta_{\tau}=Q_{u}^{v} \theta_{\tau}$. The shifted process $\tilde{X}_{t}=X_{\tau+t}$ has identical laws under $P_{\mu}^{v}$ and $Q_{u}^{\nu}$.

We write $\left(X_{t}, P\right) \cong\left(X_{t}^{\prime}, P^{\prime}\right)$ if stochastic processes $\left(X_{t}, P\right)$ and $\left(X_{t}^{\prime}, P^{\prime}\right)$ have identical laws (i.e., if they have the same finite-dimensional distributions).

3.3. Formula

$$
\hat{p}_{t}(x, d y)=q_{-t}(d y, x)
$$

determines a stationary forward transition function which is in weak duality with $p_{t}(x, d y)$ in the sense of Getoor and Sharpe [3]. We note that $\left(\tilde{X}_{-t}, Q_{u^{*}}^{\nu^{*}}\right) \cong\left(X_{t}, \hat{P}_{\nu}^{u}\right)$ where an entrance rule $\nu$ for $\hat{p}$ and an entrance rule $\nu^{*}$ for $q$ are related by the formula $\left(\nu^{*}\right)^{t}=\nu_{-t}$. Analogously, $\left(u^{*}\right)_{t}=u^{-t}$. We can assume without any substantial loss of generality that there exists a measurable transformation $r$ of the space $(\Omega, \mathscr{F})$ such that $X_{t}(r \omega)=X_{-t}(\omega)$. If $\tau$ is a stationary time, then so is $\tau^{*}(\omega)=$ $-\tau(\omega)$. Theorem 3 implies

THEOREM 4. Let stationary transition functions $p$ and $\hat{p}$ be in weak duality relative to $m$. Let $\mu$ be an entrance rule for $p$ and $\nu$ be an entrance rule for $\hat{p}$. Suppose that $\bar{\mu}$ and $\bar{\nu}$ are $\sigma$-finite and absolutely continuous with respect to $m$. Let $u$ and $v$ be excessive functions (for $p$ and $\hat{p}$ respectively) such that $\bar{\mu}=u \circ m, \bar{\nu}=v \circ m$. Then, for every stationary time $\tau,\left(X_{\tau-t}, P_{\mu}^{v}\right) \cong\left(X_{\tau^{*}+t}, \hat{P}_{\nu}^{u}\right)$. 
This is a generalization of a theorem of Getoor and Glover (see [2, Theorem (6.5)]) who have considered the situation when $\tau=\beta$,

$$
\begin{array}{ll}
\mu_{t}=\mu_{0} P_{t} & \text { for } t>0, \\
\nu_{t}=\nu_{0} \hat{P}_{t} & \text { for } t>0, \\
\mu_{t}=\nu_{t}=0 & \text { for } t \leqslant 0 .
\end{array}
$$

In this situation $\beta^{*}=\alpha=0 \hat{P}_{\nu}^{u}$-a.s. and $\left(X_{\beta-t}, P_{\mu}^{v}\right) \cong\left(X_{t}, \hat{P}_{\nu}^{u}\right)$. Moreover

$$
u=d\left(\mu_{0} G\right) / d m, \quad v=d\left(\nu_{0} \hat{G}\right) / d m
$$

where

$$
G=\int T_{t} d t, \quad \hat{G}=\int \hat{T}_{t} d t
$$

(Actually, in [2] the process $X_{(\beta-t)+}$ rather than $X_{\beta-t}$ is considered and therefore certain regularity conditions for paths are required.)

3.4. We say that a random time $\tau$ is a renewal time for a Markov process $\left(X_{t}, P\right)$ if $\left(\tilde{X}_{t}, P\right)$ where $\tilde{X}_{t}$ is defined by formula $(2.5)$ has the same transition function as $\left(X_{t}, P\right)$. If $\left(X_{t}, P\right)$ is a strong Markov process with a stationary transition function, then every optional time is a renewal time.

Markov process $\left(X_{t}, P_{\nu}^{u}\right)$ corresponding by Kuznetsov's theorem to a stationary transition function $p$, an entrance rule $\nu$ and an excessive function $u$ has the stationary transition function

$$
\begin{aligned}
& p_{t}^{u}(x, d y)=u(x)^{-1} p_{t}(x, d y) u(y) \text { for } 0<u(x)<\infty, \\
& p_{t}^{u}(x, B)=1_{B}(x) \text { for } u(x)=0 \text { or } \infty .
\end{aligned}
$$

We call $p^{u}$ the $u$-transform of $p$.

The following result is an immediate implication of Theorem 4:

THEOREM 5. Let $p, \hat{p}, \mu, \nu, u, v$ have the same meaning as in Theorem 4. If a stationary time $\tau$ is a renewal time for $\left(X_{t}, \hat{P}_{\nu}^{u}\right)$ then $\left(X_{\tau-t}, P_{\mu}^{v}\right)$ is a Markov process with a stationary transition function $\hat{p}^{v}$ (the v-transform of $\hat{p}$ ).

Theorem 5 is a generalization of Nagasawa's theorem [6].

4. A property of invariant measures.

4.1. Let $P$ be an invariant measure for a flow $\theta_{t}$. For every positive measurable $Y$ we put

$$
\bar{Y}=\int_{\mathbf{R}} \theta_{t} Y d t
$$

We note that if $\tau$ is a stationary time, then

$$
\begin{aligned}
P \kappa_{\tau} Y & =P Y \int \theta_{t} \rho(\tau) d t=\int P \theta_{t}\left(\theta_{-t} Y \rho(\tau)\right) d t \\
& =P \int \theta_{-t} Y \rho(\tau) d t=P \bar{Y} \rho(\tau)
\end{aligned}
$$

(cf. (1.1)). 
4.2. An invariant measure $P$ is called dissipative if $\bar{Y}<\infty P$-a.e. for every positive $P$-integrable $Y$, and it is called conservative if $\bar{Y}=0$ or $\infty P$-a.e. for every positive measurable $Y$. It is well known (see, e.g., [7, §V.5]) that every $\sigma$-finite invariant measure $P$ can be represented as the sum of a dissipative measure $P_{d}$ and a conservative measure $P_{c}$ which are mutually singular.

THEOREM 6. Let $P$ be a dissipative invariant measure. If $\bar{Y}_{1}=\bar{Y}_{2} P$-a.e., then $P Y_{1}=P Y_{2}$.

Indeed, let $F$ be a strictly positive $P$-integrable function. Since $\bar{F}<\infty P$-a.e., the condition (1.2) is satisfied and there exist stationary times $\tau_{a}$ such that $\kappa_{\tau_{a}} \uparrow 1$ as $a \downarrow 0$. By (4.1),

$$
P Y_{i}=\lim _{a \downarrow 0} P \bar{Y}_{i} \rho\left(\tau_{a}\right), \quad i=1,2 .
$$

4.3. Let $P$ be an arbitrary $\sigma$-finite invariant measure. There exists a partition of $\Omega$ into disjoint sets $\Omega_{c}$ and $\Omega_{d}$ such that $P_{c}\left(\Omega_{d}\right)=P_{d}\left(\Omega_{c}\right)=0$. Let $F$ be a $P$-integrable function which is strictly positive on $\Omega_{c}$ and equal to 0 on $\Omega_{d}$. We have $\bar{F}=0$ or $\infty$ $P_{c}$-a.e., $\bar{F}=0 P_{d}$ a.e. and therefore $\bar{F}=2 \bar{F} P$-a.e. On the other hand, $P(F) \neq P(2 F)$ if $P_{c} \neq 0$. Therefore the statement of Theorem 6 is true only if $P$ is dissipative.

REMARK. It follows from subsections 4.2 and 4.3 that a $\sigma$-finite invariant measure $P$ is dissipative if and only if it satisfies condition (1.2) (or (1.4)).

4.4. A measure $P_{m}^{1}$ corresponding, by Kuznetsov's theorem, to a stationary transition function $p$, an excessive measure $m$ and the exit rule $h=1$ is invariant with respect to the flow $\theta_{t}$. It is dissipative if and only if $G f<\infty, m$-a.e. where $G$ is defined in subsection 3.3.

ACKNOWLEDGEMENTS. The author is indebted to Ronald Getoor for numerous stimulating discussions.

\section{REFERENCES}

1. E. B. Dynkin, Initial and final behaviour of the trajectories of Markov processes, Uspehi Mat. Nauk 26 (160) 1971, 153-172; English transl., Markov processes and related problems of analysis, London Math. Soc. Lecture Notes Ser., vol. 54, Cambridge Univ. Press, London, 1982, pp. 123-144.

2. R. K. Getoor and J. Glover, Riesz decomposition in Markov process theory, Trans. Amer. Math. Soc. 285 (1984), 107-132.

3. R. K. Getoor and M. J. Sharpe, Naturality, standardness, and weak duality for Markov processes, Z. Wahrsch. Verw. Gebiete (to appear).

4. S. E. Kuznetsov, Construction of Markov processes with random birth and death times, Teor. Veroyatnost. i Primenen 18 (1973), 596-601. (English translation: Theory Probab. Appl.)

5. P.-A. Meyer, Probability and potential, Blaisdell, Waltham, Mass., 1966.

6. M. Nagasawa, Time reversions of Markov processes, Nagoya Math. J. 24 (1964), 177-204.

7. J. Neveu, Bases mathématiques du calcul des probabilités, Masson, Paris, 1964.

Department of Mathematics, Cornell University, Ithaca, New York 14853 (Current address)

Department of Mathematics, University of California, San Diego, la Jolla, California 92093 